\title{
Bevacizumab Induced Cardiomyopathy in a Patient with Adult Congenital Heart Disease: A Case Report and Brief Review
}

\author{
Hemalatha Narayanasamy ${ }^{1}$, Nikky Bardia ${ }^{1}$, Michael Fong ${ }^{2}$, Luanda Grazette ${ }^{2, *}$ \\ ${ }^{1}$ Keck School of Medicine of USC, Department of Medicine, Los Angles, California, USA \\ ${ }^{2}$ Division of Cardiovascular Medicine, Keck Medical Center of USC, University of Southern California, Los Angeles, California, USA
}

Email address:

grazette@usc.edu (L. Grazette)

\section{To cite this article:}

Hemalatha Narayanasamy, Nikky Bardia, Michael Fong, Luanda Grazette. Bevacizumab Induced Cardiomyopathy in a Patient with Adult Congenital Heart Disease: A Case Report and Brief Review. Journal of Cancer Treatment and Research. Vol. 3, No. 3, 2015 , pp. 32-36. doi: $10.11648 /$ j.jctr.20150303.12

\begin{abstract}
Background: Cardiac dysfunction is an unusual but documented side effect of many forms of antineoplastic therapy. Bevacizumab is a novel antineoplastic agent that inhibits VEGF-A with a low reported incidence of cardiotoxicity (1.7 - 3\%) derived primarily from clinical trials of women with breast cancer which captured adverse events of symptomatic heart failure. Subsequent studies suggest the incidence of asymptomatic decline in cardiac function may be closer to $20 \%$. Untreated declines in cardiac function are associated with increased morbidity and mortality, early recognition and treatment can improve outcomes. Case Report: Here we describe a case of a 51 year old male being treated with Bevacizumab for metastatic neuroendocrine cancer of the colon who developed a severe asymptomatic decline in cardiac function. With interruption of Bevacizumab and implementation of heart failure therapy his cardiac function returned to baseline. Conclusion: Early recognition and application of heart failure disease modifying therapy can decrease the risk and improve the likelihood of recovery in cases of chemotherapy induced cardiac dysfunction. Baseline cardiac testing and standardized surveillance can increase the opportunities for early intervention and may improve long term outcomes.
\end{abstract}

Keywords: Heart Failure, Chemotherapy, Cardiotoxic Agents, Bevacizumab, Cardiac Remodeling, Ventricular

\section{Introduction}

Vascular endothelial growth factor A (VEGF-A), is a signal protein whose release by cells stimulates vasculogenesis and angiogenesis. Overexpression of VEGF-A can contribute to the development of cancers and other diseases, such as macular degeneration. Bevacizumab is a novel antineoplastic agent that inhibits VEGF- A. It is FDA approved as part of combination chemotherapy for metastatic colorectal, non-small cell lung, and renal cell cancer (1). Major adverse events associated with bevacizumab include hypertension, increased risk of bleeding and arterial thrombosis. Although heart failure (HF) did not emerge as an increased adverse event in the early randomized placebo controlled trials an apparent increased risk of HF has been reported in several trials involving adjuvant treatment of breast cancer. Here we describe a case of a male being treated with Bevacizumab for metastatic neuroendocrine cancer of the colon who developed a severe decline in cardiac function. A brief review of bevacizumab in association with cardiac dysfunction follows the case report.

\section{Case Report}

The patient is a 51 year old male with a history of atrial septal defect repair at age 5 . At age 15 he was diagnosed with paroxysmal atrial tachyarrhythmia (primarily atrial flutter) thought to be secondary to incisional reentry. He developed a small bowel perforation at age 38 , was diagnosed with a carcinoid tumor and underwent resection which was presumed curative. Octreotide was initiated 6 years later for local recurrence and 2 years after he was diagnosed with metastasis. He was enrolled in a clinical trial and randomized to receive bevacizumab $(15 \mathrm{mg} / \mathrm{kg})$ and Octreotide. His initial course was complicated by hypertension, a previously described ADR of bevacizumab. His hypertension was controlled with 
amlodipine/olmesartan combination. A year after, on monthly antineoplastic therapy the patient noted increased frequency and duration of symptomatic atrial arrhythmias. An echocardiogram was obtained which showed normal LV size but moderately depressed function with a left ventricular ejection fraction (LVEF) of $35 \%$. There was no baseline cardiac function measured prior to initiation of therapy and there was no previous evidence or clinical history suggestive of reduced systolic function. The patient later developed permanent atrial flutter which was rate controlled with beta blockade. Extended rhythm monitoring over 10 days confirmed atrial flutter with an average rate of 80 with no significant bursts or pauses. Elective cardioversion was considered and deferred due to concerns of increased bleeding risk on anticoagulation in the setting of ongoing bevacizumab treatment. An ACE inhibitor was added to his regimen and the amlodipine/olmesartan combination was discontinued. A repeat echo one month later demonstrated severe global hypokinesis with LVEF measured at less than $20 \%$. The right ventricle, which had been normal in size and function on the initial echocardiogram, was found to be severely enlarged with mildly depressed systolic function. Cardiac MRI, less than 2 weeks later confirmed dilated cardiomyopathy with right and left ventricular global hypokinesis and LVEF estimated between $11 \%$ and $15 \%$. There was no definitive evidence of late gadolinium enhancement; however the exam was equivocal due to poor myocardial nulling. A regadenoson scintographic perfusion study showed no evidence of reversible ischemia or scar. Despite the precipitous decline in ventricular function the patient never demonstrated symptoms or signs of congestion and his BNP were only modestly elevated at $163 \mathrm{pg} / \mathrm{ml}$. However Serum galectin-3 was significantly elevated at $51 \mathrm{ng} / \mathrm{ml}$.

An aldosterone antagonist was added to his cardiac regimen and bevacizumab therapy was interrupted. Approximately 3 months after discontinuation of bevacizumab (patient remained on Octreotide) cardiac echo demonstrated normal LV and RV size with improved LVEF to $32 \%$. He remained in atrial flutter. Although re-challenge with bevacizumab was discussed the patient opted to participate in a trial involving another mode of therapy.

\section{Discussion}

\subsection{Chemotherapy Induced cardiomyopathy}

The incidence of chemotherapy induced cardiomyopathy has increased due to multiple factors including more widespread use of chemotherapy in adjuvant settings, rising number of cancer survivors resulting from increased rates of disease free survival and cure. A wide range of chemotherapeutic agents have been associated with cardiotoxicity. The anthracyclines have historically been and remain the most frequently cited and the most cardio-destructive of all antineoplastics (2). The introduction of "targeted" therapy involving monoclonal antibodies and small molecule inhibitors of protein kinases, has also contributed to the rise in incidence. These agents generally function by directly or indirectly inhibiting activity of a broad variety of growth and survival factors. Targeted anti-tumor drugs have transformed therapy and increased tumor free survival for several forms of cancer $(1,3)$. Many targeted chemotherapeutics, including agents such as imatinib, trastuzumab and sunitinib also have a demonstrable potential to interfere with normal intrinsic cardiac cellular function and viability. These effects may account for the observed higher incidence of HF amongst treated patients (4-6). Although many tyrosine kinase inhibitors have been implicated in cardiomyopathy, through a variety of mechanisms, the precise causal relationships have not been fully elucidated. This is also the case with bevacizumab where the mechanisms producing toxicity are net yet completely defined.

\section{2. $V E G F$}

The presence of VEGF and its importance in oncogenesis was first postulated in 1971 by Judah Folkman who made the salient observation that "the growth of solid neoplasm is always accompanied by neovascularization"(7). The canonical protein, now known as VEGF-A, was isolated and characterized in 1989 (8) and later identified to be identical to the previously described vascular permeability factor (9). VEGF-A has roles in angiogenesis, mitogenesis, vasculogenesis and inflammation $(2,10)$ It's over expression has been causally associated with multiple cancers (1). Numerous studies have shown decreased survival and disease-free survival in subjects with tumors over expressing VEGF (13).

\subsection{VEGF-A Signaling in the Heart}

VEGF-A plays an important role in maintaining myocardial capillary density and is active in the cardiac stress response by inducing vasodilatation, increasing nitric oxide production and endothelial cell proliferation $(11,12)$. A mouse model with cardiomyocyte specific deletion of the VEGF gene demonstrated hearts with fewer coronary micro vessels, thinned ventricular walls, cardiac fibrosis and eventual decompensated heart failure when subjected to the stress of pressure overload (13). Multiple lines of evidence suggest VEGF-A inhibition may alter intrinsic adaptive responses in the heart and thus make myocytes and other constitutive cells more vulnerable to injury (14).

\subsection{Bevacizumab}

Bevacizumab, a humanized monoclonal immunoglobulin G1 antibody, was the first approved anti-VEGF drug (15). It functions by binding to and inhibiting the activity of vascular endothelial growth factor A (VEGF-A) which in turn produces tumor inhibition through deprivation of oxygen and nutrients (15). Bevacizumab is approved as a therapeutic agent for metastatic colorectal, non-small cell lung and renal cell cancer (1). Although a provisional approval was also granted for use in the treatment of breast cancer it was later revoked due to a lack of proven survival benefit in the face of potentially 
increased risk (9).

In clinical trials, the most frequently reported Bevacizumab-related adverse events include hypertension, proteinuria, thromboembolic events, and hemorrhage. Although an increased incidence of heart failure did not emerge in the initial registrational trial, imbalances have been observed in several trials in the post registrational setting $(16,17)$.

In a retrospective meta-analysis Choueiri et al summarized the results from 5 randomized phase III clinical trials that used bevacizumab alone or in combination with other chemotherapy in the treatment of breast cancer. Un-adjudicated adverse event data of 3,784 patients were summarized (16). The results state that patients who received bevacizumab had a $4.74 \mathrm{RR}$ of developing grade III (symptomatic CHF responsive to therapy) or worse $\mathrm{CHF}$ versus those who did not $(95 \% \mathrm{CI}, 1.84$ to $12.19 ; \mathrm{P}<001)$. The summarized studies likely underestimated the actual incidence since none involved prospective cardiac monitoring and instead relied on investigator recognition of clinical CHF, a challenging proposition since the nonspecific symptoms of $\mathrm{CHF}$ and malignancy often overlap. Indeed in a prospective, single-arm phase II study of 73 newly diagnosed patients with diffuse large B-cell lymphoma (DLBCL), Avastin plus R-CHOP (rituximab, cyclophosphamide, doxorubicin, vincristine, and prednisone) where cardiac monitoring was instituted. A grade 2 or greater decline was observed in nearly $20 \%$ of patients (18). Recently, in an effort to clarify the risk of heart failure with bevacizumab Qi et al performed a meta-analysis of 19 RCTs including 16,962 patients with a variety of different tumor types (19). Based on their analysis the use of bevacizumab significantly increased the risk of developing high-grade CHF in cancer patients (RR $1.98,95 \%$ CI $1.30-3.02, \mathrm{p}=0.002)$.

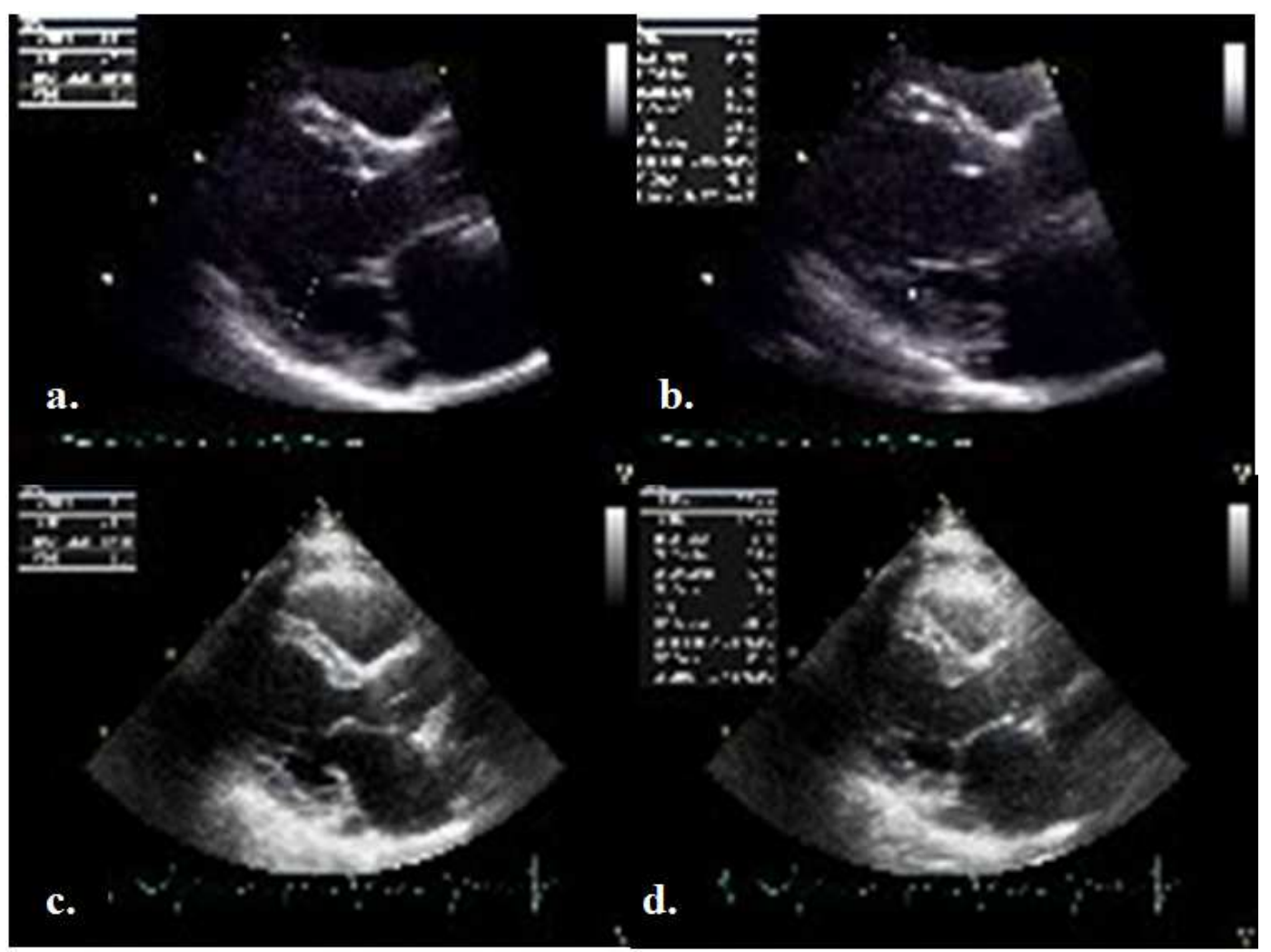

Figure 1. Left ventricular dimensions returned to normal range and ejection fraction improved after bevacizumab withdrawal and treatment with guideline directed medical therapy. a. LVIDd $5.7 \mathrm{~cm}(\mathrm{nl}=4.2-5.9 \mathrm{~cm})$ at presentation. $b$. LVIDs $4.5(\mathrm{nl}=2.1-4)$ at presentation c. LVIDd $5 \mathrm{~cm}$ after withdrawal and treatment. d. LVIDs $4.1 \mathrm{~cm}$ after withdrawal and treatment.

The results of several preclinical investigations may be interpreted to suggest that VEGF-A associated cardiac dysfunction is the result of myocardial hibernation rather than myocardial destruction (20). Indeed clinical data suggests that chemo (non-anthracycline) induced cardiomyopathy is generally responsive to treatment with heart failure therapy and frequently reversible with drug discontinuation (21). Even so it is important to recall that longstanding hibernation may be associated with progressive cellular damage and heart failure and risks should therefore not be minimized (22). Moreover in another trial of patients with DLBCL randomized to treatment with R-CHOP $(\mathrm{n}=332)$ or Avastin plus R-CHOP $(n=403)$ the incidence of CHF and decline in left-ventricular ejection fraction was significantly increased (10.9\% vs. 5.0\%). The incidence of a LVEF event, (defined as a decline from baseline of $20 \%$ for baseline LVEF $>50 \%$ or $10 \%$ decline in patients with baseline LVEF $<50 \%$ ) was also greater in the Avastin plus R-CHOP arm (10.4\%) compared to the R-CHOP alone (5.0\%). While dysfunction resolved in $82 \%$ the R-CHOP arm resolution occurred in only $62 \%$ of the Avastin plus $\mathrm{R}-\mathrm{CHOP}$ arm. The long-term cardiac consequences of VEGF inhibition is yet unknown. 


\section{Discussion}

The patient in this case had a prior history of corrected ASD and rare paroxysmal atrial tachyarrhythmia. He had no prior history of CHF, hypertension, diabetes or anthracycline exposure and the evaluation for coronary ischemia was negative. It must be noted since the earliest documented cardiac function was occurred only after therapy had been initiated and his true baseline is not known. Given the possibility of underlying systolic dysfunction and his known tachyarrhythmia it is reasonable to speculate that either or both insults may have left him with increased vulnerability to cardiac injury. The continued precipitous fall in his EF, in the absence of other inciting factors, such as ischemia or ongoing tachyarrhythmia argues plausibly for a significant contributing role for bevacizumab; as does the rapid recovery following discontinuation of the drug. Indeed the facts of the case merit a "probable" grade by both the WHO-UMC and Naranjo methods of causality assessment $(23,24)$. Interestingly despite the severity of the cardiomyopathy, the patient exhibited good functional status throughout and in fact achieved $85 \%$ of the predicted in a six minute walk test. It is unsettling to note that in the absence of symptomatic heart failure it is unlikely that his case would have been captured in a clinical trial or in routine clinical practice. Aside from those strategies used for patients treated with anthracyclines or trastuzumab no cardiac screening or monitoring standards exist for patients receiving targeted chemotherapies. Baseline functional testing in all patients prior to the potentially cardiotoxic drugs followed by surveillance testing in selected higher risk individuals (eg. older age, history of systolic dysfunction or cardiac disease) may be a reasonable approach. More data is needed to assess the utility and cost effectiveness of such a strategy.

\section{Conclusion}

We report a case of a patient who was treated with bevacizumab, for metastatic neuroendocrine tumor, and developed severe systolic dysfunction which was reversible with drug cessation and heart failure disease modifying drugs. Although the reported incidence is low the true incidence may be higher as many patients may remain relatively asymptomatic, as did our patient. The early application of disease modifying drugs as in other etiologies of cardiac dysfunction, likely contributed greatly to his recovery.

Heart failure comprises the final outcome of cumulative insults to normal cardiac structure and function. Cancer patients receiving chemotherapy have an increased risk of developing cardiovascular complications and these cardiovascular complications rise is important as cancer survivorship improves. The recognized risk factors for chemotherapy induced cardiotoxicity like advanced age, history of hypertension and history of coronary artery disease were absent in this patient and in most others affected, raising the importance of general surveillance.

Untreated declines in cardiac function are associated with increased morbidity and mortality regardless of whether they are symptomatic (25). Early recognition and use of ACE inhibitors and beta blockers are associated with decreased risk and improved likelihood of recovery for many forms of HF including that caused by chemotherapeutics including anthracyclines (26). Given the benefits of early treatment standardized close monitoring can increase opportunities for early intervention and may improve long term outcomes.

\section{References}

[1] Shih T, Lindley C. Bevacizumab: an angiogenesis inhibitor for the treatment of solid malignancies. Clinical therapeutics. 2006;28(11):1779-802.

[2] Eschenhagen T, Force T, Ewer MS, et al. Cardiovascular side effects of cancer therapies: a position statement from the Heart Failure Association of the European Society of Cardiology. European journal of heart failure. 2011;13(1):1-10.

[3] Force T, Kerkela R. Cardiotoxicity of the new cancer therapeutics--mechanisms of, and approaches to, the problem. Drug discovery today. 2008;13(17-18):778-84.

[4] Grazette LP, Boecker W, Matsui T, et al. Inhibition of ErbB2 causes mitochondrial dysfunction in cardiomyocytes: implications for herceptin-induced cardiomyopathy. Journal of the American College of Cardiology. 2004;44(11):2231-8.

[5] Kerkela R, Grazette L, Yacobi R, et al. Cardiotoxicity of the cancer therapeutic agent imatinib mesylate. Nature medicine. 2006;12(8):908-16.

[6] Force T. Double-edged sword of the new cancer therapeutics. Circulation. 2012;125(17):2057-8.

[7] Folkman J. Tumor angiogenesis: therapeutic implications. The New England journal of medicine. 1971;285(21):1182-6.

[8] Ferrara N, Henzel WJ. Pituitary follicular cells secrete a novel heparin-binding growth factor specific for vascular endothelial cells. Biochemical and biophysical research communications. 1989;161(2):851-8.

[9] Senger DR, Galli SJ, Dvorak AM, et al. Tumor cells secrete a vascular permeability factor that promotes accumulation of ascites fluid. Science. 1983;219(4587):983-5.

[10] Koch S, Claesson-Welsh L. Signal transduction by vascular endothelial growth factor receptors. Cold Spring Harbor perspectives in medicine. 2012;2(7):a006502.

[11] Giordano FJ, Gerber HP, Williams SP, et al. A cardiac myocyte vascular endothelial growth factor paracrine pathway is required to maintain cardiac function. Proceedings of the National Academy of Sciences of the United States of America. 2001;98(10):5780-5.

[12] Zentilin L, Puligadda U, Lionetti V, et al. Cardiomyocyte VEGFR-1 activation by VEGF-B induces compensatory hypertrophy and preserves cardiac function after myocardial infarction. FASEB journal : official publication of the Federation of American Societies for Experimental Biology. 2010;24(5):1467-78.

[13] Izumiya Y, Shiojima I, Sato K, et al. Vascular endothelial growth factor blockade promotes the transition from compensatory cardiac hypertrophy to failure in response to pressure overload. Hypertension. 2006;47(5):887-93. 
[14] Taimeh Z, Loughran J, Birks EJ, Bolli R. Vascular endothelial growth factor in heart failure. Nature reviews Cardiology. 2013;10(9):519-30.

[15] Mukherji SK. Bevacizumab (Avastin). AJNR American journal of neuroradiology. 2010;31(2):235-6.

[16] Choueiri TK, Mayer EL, Je Y, et al. Congestive heart failure risk in patients with breast cancer treated with bevacizumab. Journal of clinical oncology : official journal of the American Society of Clinical Oncology. 2011;29(6):632-8.

[17] Chen XL, Lei YH, Liu CF, et al. Angiogenesis inhibitor bevacizumab increases the risk of ischemic heart disease associated with chemotherapy: a meta-analysis. PloS one. 2013;8(6):e66721.

[18] Stopeck AT, Unger JM, Rimsza LM, et al. A phase 2 trial of standard-dose cyclophosphamide, doxorubicin, vincristine, prednisone (CHOP) and rituximab plus bevacizumab for patients with newly diagnosed diffuse large B-cell non-Hodgkin lymphoma: SWOG 0515. Blood. 2012;120(6):1210-7.

[19] Qi WX1, Fu S, Zhang Q, Guo XM. Bevacizumab increases the risk of severe congestive heart failure in cancer patients: an up-to-date meta-analysis with a focus on different subgroups.Clin Drug Investig. 2014 Oct;34(10):681-90

[20] Williams SP, Gerber HP, Giordano FJ, et al. Dobutamine stress cine-MRI of cardiac function in the hearts of adult cardiomyocyte-specific VEGF knockout mice. Journal of magnetic resonance imaging : JMRI. 2001;14(4):374-82.
[21] Minotti G, Salvatorelli E, Menna P. Pharmacological foundations of cardio-oncology. The Journal of pharmacology and experimental therapeutics. 2010;334(1):2-8.

[22] Lionetti V, Matteucci M, Ribezzo M, et al. Regional mapping of myocardial hibernation phenotype in idiopathic end-stage dilated cardiomyopathy. Journal of cellular and molecular medicine. 2014;18(3):396-414.

[23] Naranjo CA, Busto U, Sellers EM, et al. A method for estimating the probability of adverse drug reactions. Clin Pharmacol Ther. 1981;30(2):239-45.

[24] Centre TUM. The use of the WHO-UMC system for standardised case causality assessment.

[25] J.V. McMurray JEM, Pamela N. Peterson, Barbara Riegel, Flora Sam, Lynne W., Johnson EKK, Wayne C. Levy, Frederick A. Masoudi, Patrick E. McBride, John, Drazner GCF, Stephen A. Geraci, Tamara Horwich, James L. Januzzi, Maryl R., et al. 2013 ACCF/AHA Guideline for the Management of Heart Failure: A Report of the American College of Cardiology Foundation/American Heart Association Task Force on Practice Guidelines Circulation. 2013;128:: e240-e327.

[26] Bovelli D, Plataniotis G, Roila F, Group EGW. Cardiotoxicity of chemotherapeutic agents and radiotherapy-related heart disease: ESMO Clinical Practice Guidelines. Annals of oncology : official journal of the European Society for Medical Oncology / ESMO. 2010;21 Suppl 5:v277-82. 